\title{
Nuevas ciudadanías y nueva capacidad de influencia en decisiones estratégicas
}

Novas cidadanias e nova capacidade de influência nas decisões estratégicas

New citizenship and new influence capacity in strategic decisions

Ana María Suárez Monsalve

Doctorado en Estudios Latinoamericanos por la Universidad de Chile.

Maestría en Educación por la Pontificia Universidad Javeriana.

Profesora e investigadora en la Universidad de Medellín.

E-mail: asuarez@udemedellin.edu.com

Andreia Silveira Athaydes

- Doctorado en Comunicación Organizacional por la Universidad de Málaga y la Universidade de São Paulo (USP).

- Maestría en Management y Marketing Estratégico por la Universidad Ciencias Empresariales y Sociales (Uces).

- $\quad$ Profesora e investigadora en las Faculdades Integradas de Taquara (Faccat).

- E-mail: athaydes@faccat.br 


\section{Resumen}

Basándose en la reflexión sobre la comunicación, la influencia en decisiones estratégicas y la expresión de ciudadanías en Latinoamérica, este artículo discute el escenario organizacional en su dimensión política. La metodología reflexivo-analítica utilizó un cuestionario aplicado a 1.165 profesionales de la gestión de la comunicación en 19 países. La confianza interpersonal e institucional cada vez más baja, la crisis económica y sanitaria y el debate político constante en Latinoamérica cuestionan la capacidad de influencia en decisiones estratégicas por estos profesionales.

\section{PALABRAS CLAVE: COMUNICACIÓN・CIUDADANÍAS・LATINOAMÉRICA・ORGANIZACIONES・ESTRATEGIAS.}

\section{Resumo}

A partir da reflexão sobre comunicação, influência nas decisões estratégicas e expressão da cidadania na América Latina, este artigo discute o cenário organizacional em sua dimensão política. A metodologia reflexivo-analítica utiliza o questionário aplicado a 1.165 profissionais de gestão da comunicação em 19 países latino-americanos. A confiança interpessoal e institucional cada vez menor, as crises econômica e de saúde, e o debate político constante na região questionam a capacidade de influência nas decisões estratégicas dos profissionais da comunicação.

\section{PALAVRAS-CHAVE: COMUNICAÇÃO・CIDADANIAS・AMÉRICA LATINA・ORGANIZAÇÕES・ESTRATÉGIAS.}

\section{Abstract}

This article discusses the organizational stage in its political dimension based on a reflection on communication, influence in strategic decisions, and citizenship in Latin America. The reflexive-analytical methodology uses a survey applied to 1,165 communication management professionals in 19 countries. The dwindling interpersonal and institutional trust, the economical and health crises, and the constant political debate call the influence capacity of communication professionals' strategic decisions into question. 


\section{INTRODUCCIÓN}

$\mathrm{L}$ as expresiones de inconformidad, desconfianza y reclamo de derechos de los ciudadanos en diferentes países de América Latina invitan a revisar los aportes de Norbert Lechner (2000) sobre la expresión de nuevas ciudadanías. La situación vivida en 2020, los retos para superar la crisis por la pandemia del covid-19 y las decisiones para atender tiempos de postpandemia requieren revisar los reclamos ciudadanos a las organizaciones, tanto públicas como privadas, ante los retrocesos en derechos humanos en los primeros veinte años del siglo XXI. La igualdad ante la ley, la libertad de cada persona, el respeto por los derechos de asociación y la participación en el ejercicio del poder político, así como la reivindicación del bienestar económico y social, demandan de quienes ejercen la comunicación organizacional una actuación más decidida para orientar la reconfiguración tanto económica como social de cada institución o empresa. La capacidad de influencia profesional de Dircom en América Latina se evidencia en la participación en escenarios de decisión estratégica e, incluso, en los tipos de información que proveen a quienes toman decisiones en las organizaciones.

En este artículo se discuten los resultados del Latin American Communication Monitor (LCM, 2018) que consultó a 1.165 profesionales sobre la importancia de proveer información para la toma de decisiones. LCM busca monitorear la rutina y las percepciones de los profesionales de la comunicación estratégica y las relaciones públicas en el continente sobre ciertas tendencias del sector, permitiendo la reflexión de la categoría sobre su desempeño y las necesidades de mejora. Así, en cada edición, se consulta a los profesionales sobre un conjunto de temas que se consideran relevantes para aumentar los estándares de calidad y que, por tanto, colaboran a identificar el nivel de excelencia de los departamentos y agencias de comunicación iberoamericanos (Tench et al., 2017).

\section{REFERENTES TEÓRICOS}

\section{De la ciudadanía tradicional a las nuevas expresiones de ciudadanías}

Según las ideas predominantemente económicas de finales del siglo XIX, el Estado debería proveer las condiciones esenciales para la educación de toda persona -todo hombre, según la época-, que en ejercicio de su libertad obtuviese los recursos y la educación para ser civilizado (Marshall, 1997, p.299). Esto está asociado a un tipo de igualdad básica por la pertenencia plena a una comunidad, es decir, la ciudadanía. Alfred Marshall entendía la ciudadanía, en términos del cumplimiento de una responsabilidad traducida en deberes, como una condición que crece desde el individuo, para la cual el Estado debe proveer una influencia mediante la educación. La orientación moderna hacia la igualdad social sería la última fase de la evolución de la ciudadanía después de 250 años de desarrollo del concepto que se constituye en tres partes: la ciudadanía civil, la ciudadanía política y la ciudadanía social.

La ciudadanía civil consiste en los derechos necesarios para la libertad individual -libertad física, libertad de expresión, de pensamiento y de religión, el derecho a la propiedad, a cerrar contratos válidos, y el derecho a la justicia. Este último es de una clase distinta, porque constituye el derecho a defender y hacer valer todos los derechos en términos de igualdad con, pero mediante los procedimientos legales. Esto hace que las instituciones asociadas más directamente con los derechos civiles sean los tribunales. 
La ciudadanía política se refiere al derecho a participar en el ejercicio del poder político como miembro de un cuerpo investido de autoridad política o como elector de los miembros de tal cuerpo. Las instituciones correspondientes son el Congreso 0 las corporaciones públicas y los escenarios de gobierno local.

Y la ciudadanía social es todo el espectro desde el derecho a un mínimo bienestar económico y seguridad hasta el derecho a participar del patrimonio social y a vivir la vida de un ser civilizado conforme a los estándares de la sociedad. Por tanto, las instituciones más estrechamente conectadas con estos derechos son el sistema educativo y los servicios sociales (Marshall, 1997).

Es posible resaltar que la evolución de la ciudadanía se dio por procesos de implicaciones tanto geográficas como funcionales. Geográficas, porque se pasó de la garantía de derechos del individuo desde las costumbres de una localidad a la definición a partir de la perspectiva nacional. También por la separación de las instituciones que debían atender los tres elementos de la ciudadanía, civil, política y social. La funcionalidad, entonces, dependía tanto del alcance, de los tecnicismos y los procedimientos como del cambio de pertenencia a una comunidad de región o pueblo para considerar la dimensión nacional. Cada una de las dimensiones, entonces, desarrolló un camino de funcionalidad y atención, generando de cierta manera una división para llevarlos a la práctica real:

[...] Tras separarse, los tres elementos de la ciudadanía, enseguida perdieron el contacto, por decirlo coloquialmente. El divorcio entre ellos se consumó hasta tal punto que, sin forzar demasiado la precisión histórica, es posible asignar el período formativo en la vida de cada uno de ellos a un siglo diferente -los derechos civiles, al siglo XVIII; los políticos, al siglo XIX, y los sociales, al siglo XX. (Marshall, 1997, p.304)

En América Latina se ha desarrollado un modelo de tensión entre el Poder Ejecutivo y el Legislativo, donde ambos poderes reclaman legitimidad popular. También, las clases empresariales intervienen activamente en la política, procuran el favorecimiento de la economía con prácticas de influencia fuera de los estándares normativos y producen efectos desestabilizadores. Así, otro efecto de estas expresiones es la construcción de un imaginario colectivo de desconfianza e inseguridad respecto de sus derechos:

[...] Esto se relaciona con lo que [Guillermo] 0`Donnell ha llamado "una democracia de ciudadanía de baja intensidad" que ocurre cuando el Estado es incapaz de asegurar el imperio de la legalidad, no tanto en el área de los derechos políticos, como en el área de los derechos civiles. (O'Donnell, 1993 apud Larraín, 2005, p.46)

En consecuencia, la sociedad civil en América Latina es débil y no alcanza a expresarse completamente, además de su vulnerabilidad hacia las políticas estatales y, desde finales del siglo XX, hacia las influencias que el mercado ejerce sobre la política.

Estos mismos procesos sociales contemporáneos han creado nuevos problemas y situaciones, como los conflictos fronterizos y la trasnacionalización de estos, la alta movilidad de grupos poblacionales por migraciones, así como también las posibilidades de viaje y turismo, que ratifican la idea de que la ciudadanía evoluciona a la par con estas dinámicas, y su desarrollo va más allá de la dimensión social. Esto, entonces, ha derivado la conceptualización de nuevas ciudadanías.

\section{Nuevas ciudadanías en Latinoamérica}

A principios del siglo XXI, Norbert Lechner (2000) planteaba cómo se venían presentando procesos de transformación en la política en América Latina, que afectaban tanto las estructuras socioeconómicas como la subjetividad de la gente. Según explicaba, la pérdida de centralidad de la política ha producido también una resignificación de la ciudadanía, y para 
comprenderla hay que tomar en cuenta, por un lado, los cambios estructurales ocasionados por los procesos de diferenciación y de globalización, y por otro, los cambios en la dimensión simbólica de la política (Lechner, 2000). Los cambios estructurales están dados porque los sistemas funcionales de la sociedad, desde la economía, la educación y el ámbito jurídico, cambiaron su dependencia centrada en la política.

La autonomía del sistema económico ha generado que este actúe como mecanismo de coordinación en reemplazo 0 asumiendo el poder ordenador de la política; la expansión del poder del mercado ha establecido nuevas reglas de juego, nuevas relaciones de cooperación e, incluso, ha replanteado las relaciones de confianza en la sociedad. A esto se suma la forma en que la globalización contemporánea ha trascendido el marco nacional de la política, y lo que antes era decidido por el Estado, se ha transformado en una forma de soberanía compartida dado el intervencionismo de instancias transnacionales en las decisiones que antes eran nacionales o locales (Lechner, 2000).

Para ciudadanas y ciudadanos, las necesidades básicas de salud, educación y empleo son asuntos concretos que esperan resolverse de manera eficiente, para eso también exigen actuación concreta del Estado. No se espera que se aborden solo en el discurso político electoral ni en el discurso público de mejoramiento gubernamental. Todo lo contrario, las/los ciudadanas/ os esperan que sean asuntos abordados desde la propia subjetividad e individualidad que concentra su problemática. Pero la desconfianza, la apatía y la indiferencia, producto de esa falta de valor simbólico integrador de la política, hacen que la ciudadanía se aparte, se distancie y, en su mundo privado, da la espalda al sistema político (Lechner, 2000).

Entonces, si el poder integrador de la política y la eficiencia del Estado fallan en resolver los problemas concretos de la población, ese poder de solución y de transformación se traslada como demanda de la ciudadanía a las organizaciones empresariales que han asumido ese poder ordenador como actores del mercado. Las organizaciones del sector empresarial y del sector privado han estado incentivando cambios en las prioridades de funcionamiento social y político, por tanto, también deben generar acciones y respuestas para atender las necesidades colectivas que ya no cumple el Estado. Por ejemplo, si por su influencia en las corporaciones públicas el sector empresarial ha incidido en el financiamiento y las modalidades de consumo, en las condiciones de extracción de materiales, de explotación de territorios para insumos productivos, también deben incidir en el desarrollo sostenible, recomendar políticas e influenciar las decisiones con el fin de equilibrar los incentivos para el sector privado con las demandas de la ciudadanía, que al final debe ser la línea de los objetivos del sector público, incluso mediante normativas para que el sector privado adopte reglamentariamente prácticas sostenibles y fomenten la inversión limpia a largo plazo. Pero, el desequilibrio a favor de los intereses privados todavía permanece y genera mayor descrédito de la política y de los actores políticos.

\section{La región del mundo con menos percepción de confianza}

En este segmento se retoma el Informe 2018 del Latinobarómetro que expone cómo el apoyo a la democracia declina de manera sistemática en los países de la región hasta llegar a un 48\% de apoyo, cuando en 1997 era de un 63\%. La ola de protestas que se suscitaron desde 2010 ha generado un pensamiento consciente acerca de esta situación crítica en las democracias latinoamericanas. Además, cada año se incrementa la declaración explícita de indiferencia frente a la democracia (del 16\% en 2010 al 28\% en 2018) y, de manera clara, se observa ese alejamiento de la política, la escasa participación en partidos políticos y el descrédito por la efectividad del derecho al voto, es decir, la ciudadanía política en decadencia. Esto constituye una ciudadanía del desencanto y la frustración según el Latinobarómetro 2018 (p.14).

Esta misma corporación evalúa en sus informes la confianza en América Latina que se mide desde la confianza interpersonal hasta la confianza en las instituciones. En general, "somos la región del mundo más desconfiada de la tierra" (Latinobarómetro, 2018, p.46). En relación a la confianza interpersonal, los registros más bajos estaban en Brasil (4\%), Venezuela (8\%) y Costa Rica (10\%). 
En cambio, los que en ese mismo año presentaban más confianza interpersonal eran Colombia, Uruguay y Guatemala con el $20 \%$, respectivamente.

En cuanto a la confianza en las instituciones, esta ha disminuido progresivamente, sin embargo, se mantiene la confianza en la iglesia (63\%) a pesar de los escándalos por pedofilia y de desfalco financiero en el Vaticano, y la dudosa financiación de otras comunidades religiosas. También disminuyó la confianza en las fuerzas armadas (44\%) y en la policía (33\%).

No obstante, en cuanto a las instituciones de la democracia, se confía menos, por ejemplo, en las instituciones electorales (28\%), en el Poder Judicial (24\%), en los gobiernos (22\%), en el congreso (21\%) y en los partidos políticos (13\%) (Latinobarómetro, 2018, p.49-51). Estas instituciones son las que garantizan, en estados democráticos, la expresión de las ciudadanías civil y política.

\section{La función política de la comunicación organizacional}

En esta función es importante recurrir a los textos clásicos para revisar los orígenes, la evolución y las tendencias de la profesión. En este sentido, se revisa el valioso y orientador libro de Roberto Porto Simões (1995), Relações Públicas: função política, que en el capítulo 2, "A dimensão da micropolítica", justificó esta óptica en el tema de las relaciones públicas porque reconoce que su esencia es la relación de poder entre la organización y sus públicos, que a su vez son dos componentes del sistema social (p.35). Si bien es tradicional el estudio de la política a partir del Estado, los conflictos y las negociaciones en instituciones educativas, religiosas e industriales, bien sabemos que las políticas y normativas de algunas organizaciones son decisiones que integran y dividen, cuestionan valores o los afianzan en las tensiones constantes que da la libertad de expresión y de acción de las personas. Esas tensiones también son constantes en la relación de las organizaciones cuando entran en choque o se expresan disensos con el entorno de la macropolítica en el que está actuando el contexto económico, político, ambiental y cultural en el país y en el ámbito internacional.

Tal y como cita Porto Simões (1985), el concepto político se ha entendido como parte de la praxis humana ligado al poder, entendido como dominio y ventaja sobre los otros. Sin embargo, los micro conflictos en las organizaciones también están vinculados a las posiciones individuales o de pequeños grupos frente al trabajo, al salario, a los horarios y a las actuaciones de la organización con el entorno. Esto permite considerar los temas que atañen en la relación micro y macro política, en la cual interviene la comunicación organizacional. Por eso, es necesario recordar que:

\footnotetext{
Toda organização, seja ela qual for, além dos seus objetivos específicos (económicos, culturais, religiosos, políticos) é um subsistema social no interior da sociedade global. Enquanto subsistema social possui, em seu interior, relações sociais estáveis (interação entre indivíduos e grupos) e também um tipo particular de relação social. Nessa perspectiva, as relações políticas não designam um novo tipo de relação, mas aspectos específicos das relações sociais. Existem relações políticas e sistemas políticos na medida em que existir: 1) luta pelo poder; 2) tomada de decisão e, 3) processo de escolha. (Trindade, 1974 apudPorto Simões, 1985, p.37)
}

Assim, nesta luta de poder, as relações públicas "buscam a cooperação dos atores, possibilitando a consecução da missão organizacional, sustentada na satisfação dos interesses das partes". (Porto Simões, 2001, p.18)

Actualmente, además de los objetivos referidos a la economía, la cultura, la religión y la política se reclama, por parte de la ciudadanía, mayor claridad, decisión y actuación de las organizaciones-de todo tipo-frente a problemáticas globales. Los Objetivos de Desarrollo Sostenible concertados en Naciones Unidas (Objetivos..., 2015) así lo expresan y lo reclaman para el bien de la humanidad. Por eso, no es concebible dar la espalda a los problemas comunes; cada organización como subsistema social tiene relaciones políticas en la medida en que actúa en torno a luchas por el poder, toma de decisiones y procesos de elección. 
Moscol y Gamero (2013) han analizado la confianza en el entorno de las relaciones organizacionales y plantean esta virtud en la toma de decisiones. Los autores aclaran que la confianza se constituye desde un origen interno y, en segundo lugar, esta da la razón para la confianza externa: "La confianza se apoya en la puesta en práctica de todas las virtudes necesarias en la toma de decisiones. La consecuencia de este comportamiento ético es la construcción de confianza en quienes componen la organización y en quienes se acercan a ella" (Moscol; Gamero, 2013, p.118). Así, concluyen que se ha de entender la confianza como virtud que se necesita en los procesos para la toma de decisiones que conducen a resultados positivos.

Se ha fundamentado en la literatura de la gestión estratégica de la comunicación que el monitoreo de la reputación debe contribuir a revisar la autenticidad e integración de los discursos de la organización, interna y externamente, por tanto, las exigencias de las audiencias deben ser tema de análisis y discusión. Las analíticas de medios digitales miden la empatía, pero también pueden orientar sobre riesgos en la coherencia organizacional.

\section{Dimensión política en las organizaciones}

Como lo proponen Arancibia Carrizo y Salinas Muñoz (2016), es necesario cuestionar la función institucionalizadora de la comunicación en el orden neoliberal y discutir su carácter normalizador e integrador. Esto se puede hacer a través de las nuevas tecnologías de la información y comunicación o del rol de los medios de información en los consensos y disensos democráticos, o en las tensiones entre el saber, el poder y las relaciones que se construyen -0 destruyenen las organizaciones.

Cuando se empezó a hablar de responsabilidad social de las organizaciones empresariales en el siglo pasado, generaba incredulidad y sospecha por la conocida incompatibilidad de una actuación moral entre las ganancias, competencia por el mercado y sobrevivencia económica. Sin embargo, como plantean Arancibia Carrizo y Salinas Muñoz (2016, p.239), es por la exigencia de las personas y de la organización social, que reclama y conduce a la expedición de normas claras, que se logra convertir la utopía en realidad: "Lograr que la práctica de la Responsabilidad Social Empresarial se instale como una política consistente de las empresas, sean estas grandes, medianas o chicas, significa que la conciencia ciudadana de los individuos ha crecido y que no hay lugar para que existan relaciones de corrupción, oportunismo e inmoralidad". Y agregan que, en la responsabilidad social como principio y actuación, se logra fortalecer la conciencia ciudadana; esta debe promoverse y apoyarse desde la gestión de la comunicación en la dimensión política de las organizaciones.

Se destaca, entonces, el potencial de transformación del sector empresarial y del sector privado en general, pues orientado por la responsabilidad y el fomento de la conciencia ciudadana puede acatar y recomendar políticas que fomenten el comportamiento político y ético de ciudadanas y ciudadanos que hacen parte de sus proyectos organizativos, empezando por las directivas. Los marcos normativos de la responsabilidad no pueden ser acatados solo por los incentivos para el sector privado, para la subsistencia productiva, rentable, es imperativa la correspondencia entre el deber, el ser y el hacer en el marco de un comportamiento políticamente correcto.

\section{Capacidad de influencia política en la toma de decisiones en las organizaciones}

Las funciones de quienes trabajan en la comunicación organizacional se han estudiado y debatido entre la función operativa, la administrativa y la de asesoría estratégica. También Ferrari y França (2011) han planteado el desempeño de la función en tres dimensiones: la técnica, la gerencial y la estratégica. Para alcanzar el nivel de asesoramiento estratégico que está en el escenario de mayor vulnerabilidad y donde se trazan las grandes líneas y objetivos (Ferrari; França, 2011. p.89), se requiere, además de conocimiento, un contexto y criterio que generalmente se obtiene con una alta formación política. 
También Ferrari (2011) concluyó que grandes multinacionales son conscientes de la necesidad de una estrategia global para sus negocios y reconocen la importancia de los relacionamientos para llevarla a cabo. Según explica el autor, este tipo de organizaciones monitorean los grupos de presión, están atentas a los contextos complejos que exigen proyecciones a mediano y largo plazo, y apoyan en el análisis de escenarios para la gestión de conflictos y para enfrentar las amenazas (Ferrari; França, 2011, p.92-93).

Recientemente, en la XXVII Cátedra Unesco de Comunicación 2020 que tuvo como tema central "Pensar la comunicación, la información y los lenguajes en tiempos de pandemia", se presentó una ponencia acerca del enfoque de las capacidades para el desarrollo humano y la gestión de la comunicación aplicado al ejercicio de la profesión en un país latinoamericano (SuárezMonsalve; López Lizarazo, 2021). En los resultados de esa investigación presentados en la Cátedra, se mostró cómo el cuerpo de las capacidades con enfoque del desarrollo humano prioriza las habilidades para desempeñar la profesión con ética, compromiso, valores y persistencia, así como para generar vínculos y establecer acuerdos de manera respetuosa, empática, constructiva y colectiva (Suárez-Monsalve; López Lizarazo, 2021). Esto lleva a cómo el entorno incide en la priorización de condiciones humanas que se requiere promover y a las condiciones de adaptación según las demandas que la sociedad, la política, la cultura, el ambiente y, por supuesto, la sociedad en general reclaman de quienes se desempeñan en las organizaciones.

Los resultados de la investigación expuesta en esta Cátedra generaron cuatro categorías de capacidades para la formación y el ejercicio en gestión de la comunicación según el contexto del país, que se concentran en

capacidades desde la condición humana: ética y compromiso profesional, las capacidades referidas al análisis del contexto e incidencia en el entorno, las capacidades estratégicas relacionales y de gestión con el liderazgo de los procesos de planeación, coordinación y evaluación de la comunicación, y las capacidades para hacer frente al riesgo, referidas a las actitudes como individuo y como profesional para saber atender las crisis. (Suárez-Monsalve; López Lizarazo, 2021, 2019-2020)

Las capacidades referidas al análisis del contexto e incidencia en el entorno agrupan condiciones necesarias para observar, reflexionar, analizar y comprender el entorno e incidir sobre el mismo, como la capacidad para el pensamiento estratégico para establecer criterios de valoración y acción puntual al desarrollo de estrategias de manera holística y la capacidad lingüística, narrativa, de diálogo y deliberación. Todas estas capacidades tienen, en su esencia, el criterio político de quienes ostentan la responsabilidad de asesorar, liderar, administrar y ejecutar procesos de comunicación y relacionamiento estratégico en/para organizaciones.

Los profesionales de la comunicación enfrentan múltiples tensiones en su trabajo, producto de las variables sociales, económicas y políticas que afectan todo tipo de organizaciones. La conciliación de intereses privados y públicos, las demandas tanto de grupos de interés, de las audiencias digitales como de los públicos internos, las obligaciones legales y las urgencias ambientales, entre otros asuntos, son objeto de permanentes solicitudes a los equipos de gestión de la comunicación, ya sea in house o por el servicio de asesoría prestados por agencias consultoras externas.

Los practicantes de la comunicación de diferente función, ya sea técnica, gerencial o estratégica, se adaptan a esta tensión instrumental. Sin embargo, las exigencias de los públicos con sus múltiples intereses, las fricciones por el poder y las crisis económicas y reputacionales ponen a prueba el compromiso social y la agencia de transformación social en el rol profesional. Es a través del desarrollo de capacidades de análisis crítico, con amplia cobertura de información y datos, con el panorama histórico y prospectiva a la vez y la claridad sobre las implicaciones presentes y futuras que se logra la influencia en decisiones estratégicas en los momentos y escenarios de deliberación. La claridad sobre las oportunidades que ofrece la gestión de la comunicación para incidir en esos escenarios parte del conocimiento en el saber profesional ubicándolo en el contexto político de decisión. 


\section{METODOLOGÍA}

Los datos que se presentan parten del estudio cuantitativo estadístico usando el software SPSS, con un cuestionario de 39 preguntas que miden un conjunto de variables dicotómicas, nominales y ordinales documentadas teóricamente. Para este artículo se toman los datos de la sección "Información para apoyar la toma de decisiones", que corresponde a las preguntas del cuestionario Q7 a Q10, en correlación con las variables demográficas cuyos resultados fueron estadísticamente significativos. El cruce de variables se realiza por la significancia estadística interpretando diferentes coeficientes (chi-cuadrado, correlación de Pearson, ANOVA/Scheffe Post-hoc, test de independencia de la T de Kendall y test de correlación de Kendall). El estudio consultó en 2018' una muestra de profesionales de la gestión en comunicación ubicados en 19 países de América Latina y representa una población de más de 20.000 profesionales contactados, de los cuales 2.575 ingresaron a la encuesta autoadministrada y, finalmente, 1.165 que resolvieron completamente el cuestionario superando los filtros de validez y rigor de la investigación, completando las 39 preguntas, incluyendo datos demográficos. La muestra tiene entre 41 y 42 años en promedio, con una participación de 60,1\% de mujeres y un 39,9\% de hombres; y en cuanto a la experiencia, el 44,1\% tiene entre 6 y 10 años de experiencia, y el 55,9\% hasta 5 años.

Las preguntas que corresponden a la sección analizada en este artículo son las siguientes:

Q7: Muchos departamentos de comunicación/agencias proporcionan conocimiento a los altos directivos y clientes (internos) difundiendo información mediante resúmenes de noticias diarios, monitoreo o reporte de medios, resultados de encuestas y otros informes como escenarios o auditorías. Por favor, evalúa las siguientes afirmaciones según su experiencia. Ítem: proveer información a los que toman las decisiones no es una tarea fundamental para nuestro departamento/agencia. Escala 1 (Fuertemente en desacuerdo) - 5 (Fuertemente de acuerdo).

Q8: ¿Tu departamento/agencia provee información como resúmenes de noticias, monitoreo de medios, resultados de encuestas, informes de marca/reputación, auditoría o informes de situación a los altos ejecutivos y/o clientes (internos)?

Q9a: ¿Con qué frecuencia provee tu departamento o agencia la siguiente información al director de comunicación y/u otros clientes (internos)? Resúmenes de noticias (ej. Descripciones editadas y organizadas de noticias y debates en los medios masivos, social media, etc.); informes de monitoreo/seguimiento de medios (ej. Clippingsy evaluación de las noticias en medios masivos o social media); informes de reputación/marca (ej. Basados en la imagen o la evaluación de marca); resultados de encuestas (ej. Encuestas de empleados o clientes); informes de auditorías (ej. Comparaciones internas/externas); informes de antecedentes de temas (ej. Temas estratégicos, escenarios); informes de antedecentes de stakeholders (ej. Rivales potenciales, influencers, colaboradores)

Q9b: ¿Qué informes están principalmente preparados por servicios externos de proveedores?

Q10: ¿Con qué frecuencia tu departamento/agencia proporciona la siguiente información a sus clientes internos o externos? Monitoreo de prensa (clippings/evaluación); monitoreo de social media (clippings/evaluación); monitoreo de TV (clippings/ evaluación); resúmenes de noticias (editado/contenido valorado).

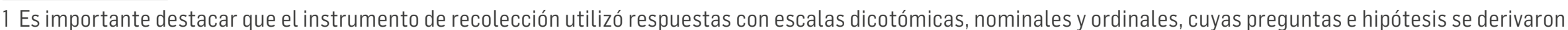

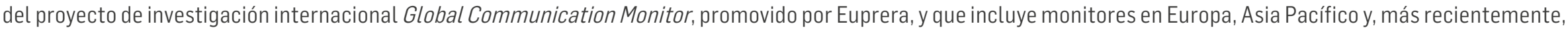
América del Norte. 


\section{ANO 18 • NÚMERO 37 • SETEMBRO / DEZEMBRO 2021 - ORGANICOM}

\section{Hallazgos de la sección "Información para apoyar la toma de decisiones"}

Los datos evidencian que en los Departamentos de Comunicación representados en el estudio con ubicación en América Latina tienen una comprensión limitada de proporcionar información para la toma de decisiones estratégicas. Seis de cada diez profesionales de la comunicación reconocen que esta función ha ganado importancia en los últimos años. Sin embargo, a pesar de que un 83\% de agencias y departamentos de comunicación de los consultados reconocen que esta es una función propia de la comunicación organizacional, un alto porcentaje no la evidencian como importante. Los informes que presentan con mayor frecuencia son los resúmenes de noticias (75\%) y los informes de monitoreo de medios (74\%).

La mayoría de los profesionales reconocen las grandes oportunidades que ofrece aportar información para la toma de decisiones. En una escala de 1 a 5 , siendo 5 el valor otorgado para estar de acuerdo, 3 un punto de vista neutral y 1-2 el valor que representa estar en desacuerdo, el 69,7\% dice que está de acuerdo en que esta función ofrece grandes oportunidades para ganar reconocimiento por parte del nivel más alto de dirección y por los clientes. Un 21,3\% es neutral y un 9\% está en desacuerdo con esta afirmación. Aun así, el 64,9\% reconoce que aportar información para la toma de decisiones está ganando relevancia para el departamento de comunicación y/o agencia; un 25\% es neutral ante esta premisa y un 10\% está en desacuerdo.

Un 73,8\% de la muestra está de acuerdo con que proveer información para las decisiones estratégicas ofrece oportunidades para posicionarse frente a otros departamentos, un 18,9\% se ubica como neutral y un 7,3\% está en desacuerdo.

En la descripción por tipo de organización, los trabajadores de empresas que cotizan en la bolsa de valores son los que más consideran que esta función está ganando relevancia, así como para agencias y consultoras. Las personas que menos están de acuerdo con esta apreciación son las que trabajan en empresas privadas. De igual manera en la valoración de esta como una gran oportunidad para ganar reconocimiento del nivel directivo y de los clientes (internos) están más de acuerdo quienes trabajan en empresas que cotizan en bolsa y quienes trabajan en consultoras y agencias.

Los informes de monitoreo, tanto realizados regularmente por las Direcciones de Comunicación como si son informes externalizados, son los tipos de contenido que más se preparan y proveen para la toma de decisiones. A estos les sigue los resúmenes de noticias. Sin embargo, hay diferencias significativas en estas apreciaciones según los tipos de organizaciones. Por ejemplo, estos resúmenes son las tareas más valoradas como información relevante para la toma de decisiones por parte de las agencias y consultoras, así como por las organizaciones gubernamentales. Llama la atención que informes de auditorías, resultados de encuestas y antecedentes de temas y de stakeholders no son muy valorados en ninguna de las organizaciones. Este tipo de informes ofrece contexto situacional y provee datos relevantes de antecedentes e implicaciones temáticas que contribuyen a entender mejor las problemáticas. Aun así, son desconocidos en su relevancia para la toma de decisiones por parte de quienes participaron en esta encuesta en 2018. En cambio, los reportes acerca de la reputación están con valores promedio en todos los tipos de organizaciones consultadas (Tablas 1 y 2).

Tabla 1: Información para apoyar la toma de decisiones - resultados por países

\begin{tabular}{c|c|c|c|c|c|c}
\hline & \multicolumn{2}{|c|}{ Resúmenes de Noticias } & \multicolumn{2}{c|}{ Informes de Monitoreo } & \multicolumn{2}{c}{ Reputación/Marca } \\
\cline { 2 - 7 } & $\begin{array}{c}\text { Regularmente } \\
(\%)\end{array}$ & $\begin{array}{c}\text { Externalizada } \\
(\%)\end{array}$ & $\begin{array}{c}\text { Regularmente } \\
(\%)\end{array}$ & $\begin{array}{c}\text { Externalizada } \\
(\%)\end{array}$ & $\begin{array}{c}\text { Regularmente } \\
(\%)\end{array}$ & $\begin{array}{c}\text { Externalizada } \\
(\%)\end{array}$ \\
\hline Argentina & 66,4 & 47,7 & 75,7 & 62,6 & 15,9 & 28 \\
\hline Brasil & 73 & 53,6 & 78,4 & 69,8 & 33,3 & 32,4 \\
\hline
\end{tabular}


Tabla 1: Continuación

\begin{tabular}{c|c|c|c|c|c|c}
\hline & \multicolumn{2}{|c|}{ Resúmenes de Noticias } & \multicolumn{2}{c|}{ Informes de Monitoreo } & \multicolumn{2}{c}{ Reputación/Marca } \\
\cline { 2 - 7 } & $\begin{array}{c}\text { Regularmente } \\
(\%)\end{array}$ & $\begin{array}{c}\text { Externalizada } \\
(\%)\end{array}$ & $\begin{array}{c}\text { Regularmente } \\
(\%)\end{array}$ & $\begin{array}{c}\text { Externalizada } \\
(\%)\end{array}$ & $\begin{array}{c}\text { Regularmente } \\
(\%)\end{array}$ & $\begin{array}{c}\text { Externalizada } \\
(\%)\end{array}$ \\
\hline Chile & 83,1 & 63,6 & 84,7 & 56,8 & 28 & 40,7 \\
\hline Colombia & 66,2 & 40 & 66,2 & 50,8 & 36,9 & 30,8 \\
\hline Costa Rica & 77,5 & 40 & 82,5 & 65 & 27,5 & 22,5 \\
\hline Ecuador & 78 & 44 & 66 & 58 & 29,8 & 36 \\
\hline México & 76,2 & 42,9 & 72,6 & 65,5 & 29,8 & 32,1 \\
\hline Perú & 71,4 & 52,4 & 54,8 & 64,3 & 40,5 & 23,8 \\
\hline República & 81,8 & 40,9 & 81,8 & 59,1 & 50 & 36,4 \\
\hline Dominicana & 77,8 & 40 & 68,9 & 53,3 & 28,9 & 35,6 \\
\hline Uruguay & 79,3 & 50 & 77,6 & 53,4 & 39,7 & 24,1 \\
\hline Venezuela & & & & & & \\
\hline
\end{tabular}

Fuente: Latin American Communication Monitor 2018-2019.

Tabla 2: Información para apoyar la toma de decisiones - resultados por países

\begin{tabular}{|c|c|c|c|c|c|c|c|c|}
\hline & \multicolumn{2}{|c|}{$\begin{array}{l}\text { Resultados de } \\
\text { encuestas }\end{array}$} & \multicolumn{2}{|c|}{ Informes de auditorías } & \multicolumn{2}{|c|}{$\begin{array}{l}\text { Informes de } \\
\text { antecedentes } \\
\text { de temas } \\
\end{array}$} & \multicolumn{2}{|c|}{$\begin{array}{c}\text { Informes de } \\
\text { antecedentes de } \\
\text { stakeholders }\end{array}$} \\
\hline & $\begin{array}{c}\text { Regularmente } \\
(\%)\end{array}$ & $\begin{array}{c}\text { Externalizada } \\
(\%)\end{array}$ & $\begin{array}{c}\text { Regularmente } \\
(\%)\end{array}$ & $\begin{array}{c}\text { Externalizada } \\
(\%)\end{array}$ & $\begin{array}{c}\text { Regularmente } \\
(\%)\end{array}$ & $\begin{array}{c}\text { Externalizada } \\
(\%)\end{array}$ & $\begin{array}{c}\text { Regularmente } \\
(\%)\end{array}$ & $\begin{array}{c}\text { Externalizada } \\
(\%)\end{array}$ \\
\hline Argentina & 29 & 49,5 & 20,6 & 18,7 & 35,5 & 8,4 & 27,1 & 11,2 \\
\hline Brasil & 33,3 & 41,4 & 23 & 17,1 & 32 & 11,3 & 26,6 & 14,4 \\
\hline Chile & 35,6 & 40,7 & 18,6 & 19,5 & 49,2 & 22 & 44,1 & 22,9 \\
\hline Colombia & 36,9 & 23,1 & 38,5 & 16,9 & 33,8 & 12,3 & 26,2 & 7,7 \\
\hline Costa Rica & 20 & 22,5 & 12,5 & 15 & 30 & 10 & 32,5 & 20 \\
\hline Ecuador & 16 & 36 & 22 & 26 & 34 & 10 & 18 & \\
\hline México & 38,1 & 41,7 & 23,8 & 13,1 & 46,4 & 8,3 & 51,2 & 10,7 \\
\hline Perú & 33,3 & 26,2 & 28,6 & 23,8 & 38,1 & 11,9 & 42,9 & 16,7 \\
\hline $\begin{array}{l}\text { República } \\
\text { Dominicana }\end{array}$ & 36,4 & 29,5 & 31,8 & 15,9 & 40,9 & 18,2 & 40,9 & 20,5 \\
\hline Uruguay & 26,7 & 35,6 & 13,3 & 31,1 & 33,3 & 4,4 & 31,1 & 8,9 \\
\hline Venezuela & 39,7 & 55,2 & 34,5 & 24,1 & 41,4 & 17,2 & 46,6 & 15,5 \\
\hline
\end{tabular}

Fuente: Latin American Communication Monitor 2018-2019. 
En cuanto a la frecuencia, los informes diarios contienen datos de monitoreo de medios escritos y resúmenes de noticias en general. Los demás medios, como televisión y las redes sociales, tienen menos frecuencia en su preparación y entrega a quienes toman decisiones. El monitoreo de social media tiene el menor porcentaje en frecuencia diaria de entrega, pero son los reportes con frecuencia semanal que más se preparan para quienes toman decisiones. Sin embargo, tal y como se muestra en la Tabla 3, la prevalencia de los tipos de informes cambia entre países. Así, mientras que el Monitoreo de prensa (clippings/evaluación) es importante para todos, en general, el monitoreo de social media tiene valores muy diferentes entre Uruguay (32,5\%) y Brasil (66,7\%), por ejemplo. 0 en el caso de los monitoreos de televisión que son más valorados en Chile $(66,1 \%)$ que en Colombia (37,3\%).

Tabla 3: Información para apoyar la toma de decisiones: aportación diaria o semanal de monitoreo y resúmenes de noticias en distintos países de Latinoamérica

\begin{tabular}{c|c|c|c|c}
\hline & $\begin{array}{c}\text { Monitoreo de } \\
\text { prensa (clippings/ } \\
\text { evaluación) \% }\end{array}$ & $\begin{array}{c}\text { Monitoreo de social } \\
\text { media (clippings/ } \\
\text { evaluación) } \%\end{array}$ & $\begin{array}{c}\text { Monitoreo de } \\
\text { TV (clippings/ } \\
\text { evaluación) \% }\end{array}$ & $\begin{array}{c}\text { Resúmenes } \\
\text { de noticias } \\
\text { (contenido editado/ } \\
\text { depurado) \% }\end{array}$ \\
\hline Argentina & 69,2 & 59,2 & 45,2 & 57,3 \\
\hline Brasil & 83,2 & 66,7 & 51,7 & 64,8 \\
\hline Chile & 85,7 & 53,6 & 66,1 & 71,7 \\
\hline Colombia & 63,8 & 55,2 & 37,3 & 49,2 \\
\hline Costa Rica & 78,9 & 63,2 & 60,5 & 76,9 \\
\hline Ecuador & 77,6 & 60,4 & 54,6 & 63,3 \\
\hline México & 63,8 & 61,3 & 51,3 & 68,3 \\
\hline Perú & 73,2 & 62,5 & 45 & 75 \\
\hline República & 75 & 66,7 & 45,2 & 60,5 \\
\hline Dominicana & 69,8 & 32,5 & 48,1 & 80,4 \\
\hline Uruguay & 84,2 & 73,2 & & \\
\hline Venezuela & & & & 75 \\
\hline
\end{tabular}

Fuente: Latin American Communication Monitor 2018-2019.

El tema de la evolución digital y la web social ha estado presente en las tres ediciones del Latin American Communication Monitor entre 2014 y 2018, y se mantuvo como el tema más importante para la gestión de la comunicación hasta 2021. Por esto, llama la atención que el monitoreo de social media haya sido un tipo de reporte con valores medios y bajos tanto en la frecuencia diaria como en el tipo de informes por tipo de organización. Como informa el LCM (Moreno et al., 2019, p.42), tanto para empresas que cotizan en bolsa como para empresa privada, para organizaciones gubernamentales y para organizaciones sin ánimo de lucro son apenas la tercera actividad diaria o semanal entre los cuatro tipos de informes que se proveen para la toma de decisiones.

\section{DISCUSIÓN}

En las discusiones conceptuales se permite pensar nuevamente en la función política de la profesión en las organizaciones y su relación con el entorno. Cumplir esa función requiere la formación de capacidades para interpretar la dinámica del entorno, analizar causas y consecuencias en la vida de las personas que la conforman. Además, se requieren habilidades para incidir en los escenarios de decisión, tanto interno como externo, capacidades para administrar la controversia, usar el diálogo para la concertación y, fundamentalmente, un compromiso ético con el bienestar común. 


\section{ANO 18 • NÚMERO 37 • SETEMBRO / DEZEMBRO 2021 - ORGANICOM}

La habilidad para la incidencia en decisiones necesita de información constante, clara y oportuna. Sin embargo, es habitual proveer información para que otras personas decidan se quedan en una función instrumental de la comunicación organizacional. Los datos muestran que aún hay trabajadores que no están totalmente convencidos de las oportunidades que ofrece brindar información para los grupos que toman decisiones. Tampoco es un consenso que los diferentes tipos de informes que realizan quienes se desempeñan en dirección o equipos de área de comunicación se constituyan en oportunidad para posicionarse ante las diferentes áreas de la organización. Las diferencias en las respuestas sobre los tipos de organización muestran que las organizaciones que cotizan en bolsa o en agencias consultoras tienen claro que estos informes brindan legitimidad como aportes desde la comunicación organizacional.

El monitoreo de los discursos sobre la organización en diferentes soportes debe ser visto como función estratégica y no solo función operativa. Independientemente del soporte, ya sea medios tradicionales o medios digitales, lo importante es identificar escenarios de vulnerabilidad, de alerta y de atención oportuna. La escucha es fundamental en los procesos de comunicación organizacional y ante escenarios turbulentos como es el contexto de los países latinoamericanos.

Es urgente y necesario entender la naturaleza del descontento y la movilización en los países de la región. América Latina vive una crisis constante y creciente de desconfianza, de reclamo con llamados de angustia y de petición de oportunidades para los grupos sociales menos favorecidos. Como se ha presentado, el Latinobarómetro descompone la expresión de desconfianza de la ciudadanía, el descrédito progresivo de las instituciones y la pérdida de interés en los mecanismos democráticos. Esto afecta indudablemente a las organizaciones como ya se ha fundamentado y muestra que las ciudadanías esperan respuesta y solución y, ante la falta de credibilidad en los gobiernos e instituciones sociales, actuación coherente de las organizaciones privadas, especialmente del sector productivo porque los problemas que hay que atender son asuntos urgentes de la vida cotidiana: pobreza, empleo, hambre, educación, acceso a la tecnología, medio ambiente. Las propuestas de solución gubernamentales tardan y se enredan en los procesos electorales, lo que ya no es alternativa para las urgencias sociales y económicas de subsistencia.

Al monitorear a los públicos en las redes sociales, en las evidencias de relacionamiento, con las auditorías de comunicación, con los mensajes y contenidos que se publican en los medios tradicionales y nuevos, se observa un análisis del entorno, ponderación de las demandas y generación de solución. La evolución digital debe servir para que este monitoreo sea una forma de interpretar demandas de ciudadanos que ejercen sus derechos desde la expresión de ciudadanías política, civil y social, así como también de nuevas expresiones de ciudadanías que han permanecido al margen de la participación. También hay ciudadanías instrumentales que reclaman, sin clasificarse en los conceptos tradicionales, y se expresan generalmente con sus propios recursos, su voz y acción. Que estas voces y acciones no tengan que recurrir a la violencia hace parte de la atención oportuna de problemas detectados antes de que estallen. Las capacidades para la lectura del entorno, la incidencia en decisiones, la deliberación y el diálogo son necesarias y se deben fomentar en la práctica cotidiana de la comunicación organizacional.

Los profesionales de la comunicación organizacional solo alcanzarán un nivel de asesoramiento estratégico a través del conocimiento profundo acerca del saber específico puesto en los contextos interno y externo en que se desempeña la organización. El criterio profesional se obtiene con una alta formación política e incluye cuestionar la función institucionalizadora de la comunicación, funcional e instrumentalizada. Así, con información, comunicación y relacionamientos estratégicos, se gestionan los disensos y los consensos en escenarios democráticos. Cabe destacar que las organizaciones son escenarios de disputa interna por el poder, además de actores en el juego de poder político nacional e internacional, para tanto, ser conscientes de que estas dimensiones son afectadas por los procesos informativos, comunicacionales y relacionales es parte del rol profesional. Actuar con consciencia frente a esa capacidad de influencia es un compromiso que supera la dimensión profesional y trasciende a la dimensión ciudadana. De esta forma, la dimensión política de las organizaciones es influenciada por la dimensión política de profesionales de la comunicación. 
Finalmente, los resultados mostraron que en los Departamentos de Comunicación en las organizaciones todavía se tiene una definición limitada sobre proporcionar información a quienes dirigen y hacen parte de escenarios de decisión. Incluso, aunque un 83\% de agencias y departamentos de comunicación están de acuerdo en que proporcionar información para las directivas ejecutivas es una función de la comunicación organizacional, aún hay profesionales en América Latina que no lo ven como una tarea fundamental de su área. Un 65\% de profesionales en comunicación opinaron que aportar información ha ganado relevancia en la organización y en las agencias. Sin embargo, los resúmenes de noticias (75\%) y los informes de monitoreo de medios (74\%) siguen siendo el tipo de información que proveen con mayor frecuencia, dejando de lado análisis más profundos y proyectivos. El monitoreo de la reputación debe contribuir a revisar la autenticidad e integración de los discursos de la organización interna y externamente. Las exigencias de las audiencias deben ser tema de discusión y esto supera el alcance de publicaciones en redes sociales. Las analíticas de medios digitales miden la empatía, pero también pueden orientar sobre riesgos en la coherencia organizacional.

\section{CONSIDERACIONES FINALES}

La toma de decisiones es un proceso que se apoya en los conocimientos de la profesión y la elección de acciones en un contexto complejo. En la gestión de la comunicación organizacional es habitual proporcionar información para quienes toman las decisiones que llevar a cabo su participación como actores y responsables de esos escenarios.

Los resultados de este estudio evidencian que los trabajadores en la gestión de la comunicación organizacional en América Latina consideran que proveer de información a los tomadores de decisiones es importante, pero no es una posición unánime. La mayoría reconoce que esta función de proporcionar información genera reconocimiento a sus labores y a su rol organizacional como profesionales.

Los departamentos de comunicación muestran una visión limitada sobre proporcionar información a quienes toman decisiones y siguen basándose fundamentalmente en el monitoreo de medios, redes sociales y en menor medida a evaluar temas estratégicos, stakeholders, redes sociales y reputación.

La consideración instrumental de proveer de información a los tomadores de decisiones organizacionales y el hecho de no hacer, en su mayoría, parte de los escenarios de decisión con voz crítica revelan que se da la espalda a oportunidades para influenciar las decisiones cumpliendo un rol como agentes de transformación social.

La desconfianza en los sistemas democráticos ha cambiado las formas de expresión de las ciudadanías. Si bien los conceptos clásicos se mantienen, en la práctica la apropiación de deberes y derechos ciudadanos ha cambiado por el descrédito, la apatía y la falta de legitimidad. Nuevas ciudadanías se expresan en diferentes escenarios y, generalmente, constituyen los últimos recursos para reclamar solución a problemas que ponen en riesgo la sobrevivencia con dignidad en los países latinoamericanos.

La función política de la comunicación en las organizaciones ofrece bases teóricas para entender la dinámica de las relaciones de poder, la toma de decisiones y la libertad de elección en la micropolítica. Estos elementos son constantes en la dinámica macropolítica, así la intervención en ambas es parte de esa función profesional para lograr consensos y reconocer disensos. Los profesionales de la comunicación organizacional están presentes en estos escenarios de tensión en las relaciones sociales desde su condición política para intervenir con justicia y libertad.

También, es importante cuestionar permanentemente la verdadera capacidad de influencia de profesionales de la comunicación organizacional en escenarios de decisión. Esto hará reaccionar a quienes, teniendo la oportunidad de acceder a estos escenarios, se mantienen en funciones operativas generando reportes de monitoreo, pero sin profundidad analítica, 
de previsión de riesgos y con prospectiva. Mantenerse en la provisión de información de seguimiento es apenas una función de nivel intermedio, operativa y administrativa, hay que trascender a la estratégica, a los estudios y análisis de contexto, de antecedentes y de proyección según los patrones de comportamiento de los stakeholders, de audiencias clave y de públicos con influencia política en las organizaciones.

El monitoreo de la reputación debe contribuir a revisar la autenticidad e integración de los discursos de la organización interna y externamente. La desconfianza de la ciudadanía se puede subsanar solo con coherencia, autenticidad y transparencia. Mantener la reputación sin cambios efectivos en la sociedad, y más en América Latina, puede constituirse solo en una distracción. Son riesgos buscados a corto plazo.

En tiempos de convulsión social, en crisis de salud y económica, y con el debate político constante en los países latinoamericanos, se requiere mayor formación política para incidir en los asuntos públicos. Es una capacidad estratégica que desarrollar entre profesionales y, más aún, un requisito como ciudadanos.

\section{REFERENCIAS}

ARANCIBIA-CARRIZO, Juan Pablo; SALINAS-MUÑOZ, Cláudio. Comunicación política y democracia en América Latina. Barcelona: Gedisa, 2016.

CÁTEDRA UNESCO DE LA COMUNICACIÓN, 28., 2020, Bogotá. Anais [...]. Bogotá: Pontificia Universidad Javeriana, 2020.

FERRARI, Maria Aparecida. A influência dos valores organizacionais na determinação da prática e do papel dos profissionais de relações públicas: estudo comparativo entre organizações do Brasil e do Chile. 2000. Tese (Doutorado em Ciências da Comunicação) - Universidade de São Paulo, São Paulo, 2000.

FERRARI, Maria Aparecida; FRANÇA, Fábio. Relaciones públicas: naturaleza, función y gestión en las organizaciones contemporáneas. Buenos Aires: La Crujía, 2011.

LARRAÍN, Jorge. ¿América Latina moderna?Globalización e identidad. Santiago: LOM, 2005.

LATIN COMMUNICATION MONITOR. Informes 2018-2019. 3.ed. Madrid: 2020. Disponível em: https://bit.ly/3aXbpvi. Acesso em: 15out. 2021.

LATINOBARÓMETRO. Informe 2018. Santiago de Chile: Latinobarómetro, 2018. Disponível em: https://bit.ly/30Lgsgl. Acesso em: 15out. 2021.

LECHNER, Norbert. Nuevas ciudadanías. Revista de Estudios Sociales, Bogotá, n.5, p.25-31, 2000. doi: https://doi.org/10.7440/ res5.2000.03.

MARSHALL, Thomas Humphrey. Ciudadanía y clase social. Revista Española de Investigaciones Sociológicas, Madrid, n.79, p.297-334, 1997.

MORENO, Ángeles; MOLLEDA, Juan Carlos; ÁLVAREZ-NOBELL, Alejandro; HERRERA, Marco; ATHAYDES, Andréia; SUÁREZ, Ana María. Comunicación estratégica y sus retos: fake news, confianza, información para la toma de decisiones, liderazgo, 
satisfacción, estrés y compromiso laboral. Resultados de una encuesta en 19 países. Bruxelles: Latin American Communication Monitor, 2019.

MOSCOL, Ronnie; GAMERO, Renato. Aproximación a la confianza como bien interno de las Relaciones Públicas. Revista de Comunicación, Piura, v.12, p.110-138, 2013.

OBJETIVOS y metas de desarrollo sostenible. Naciones Unidas, [s.l.], 2015. Disponível em: https://bit.ly/3aV7sri. Acesso em: 15out. 2021.

O'DONELL, G. On the State, democratization and some conceptual problems (a Latin American view with glances at some post-communoist countries). The Hellen Kellog Institute for International Studies, South Bend, n.192, 1993.

PORTO SIMÕES, Roberto. Relações Públicas: função política. São Paulo: Summus, 1995.

PORTO SIMÕES, Roberto. Relações públicas e micropolítica. São Paulo: Summus, 2001.

SUÁREZ-MONSALVE, Ana María; LÓPEZ LIZARAZO, Carlos Alfonso. Aportes para discutir el enfoque de las capacidades en la gestión de comunicación. Aplicación en Colombia. Revista Comunicacao, Mídia e Consumo, São Paulo, v.18, n.53, p.200-222, 2021.

TENCH, Ralph; VERČIČ, Dejan; ZERFASS, Ansgar; MORENO, Angeles; ERHOEVEN, Piet. Communication excellence. How to develop, manage and lead exceptional communications. Cham: Palgrave Macmillan, 2017.

Artículo recibido el 30.06.2021 y aprobado el 27.09.2021. 